\title{
Crystal Structures of the Wild Type and the Glu376Gly/Thr255Glu Mutant of Human Medium-Chain Acyl-CoA Dehydrogenase: Influence of the Location of the Catalytic Base on Substrate Specificity ${ }^{\dagger}$
}

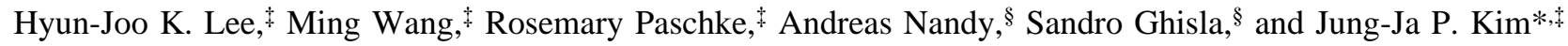 \\ Department of Biochemistry, Medical College of Wisconsin, Milwaukee, Wisconsin 53226, and Faculty of Biology, \\ University of Konstanz, P.O. Box 5560-M644, D-78434 Konstanz, Germany
}

\begin{abstract}
Crystal structures of the wild type human medium-chain acyl-CoA dehydrogenase (MCADH) and a double mutant in which its active center base-arrangement has been altered to that of long chain acyl-CoA dehydrogenase (LCADH), Glu376Gly/Thr255Glu, have been determined by X-ray crystallography at 2.75 and $2.4 \AA$ resolution, respectively. The catalytic base responsible for the $\alpha$-proton abstraction from the thioester substrate is Glu376 in MCADH, while that in LCADH is Glu255 (MCADH numbering), located over 100 residues away in its primary amino acid sequence. The structures of the mutant complexed with C8-, C12, and C14-CoA have also been determined. The human enzyme structure is essentially the same as that of the pig enzyme. The structure of the mutant is unchanged upon ligand binding except for the conformations of a few side chains in the active site cavity. The substrate with chain length longer than $\mathrm{C} 12$ binds to the enzyme in multiple conformations at its $\omega$-end. Glu255 has two conformations, "active" and "resting" forms, with the latter apparently stabilized by forming a hydrogen bond with Glu99. Both the direction in which Glu255 approaches the $\mathrm{C}_{\alpha}$ atom of the substrate and the distance between the Glu255 carboxylate and the $\mathrm{C}_{\alpha}$ atom are different from those of Glu376; these factors are responsible for the intrinsic differences in the kinetic properties as well as the substrate specificity. Solvent accessible space at the "midsection" of the active site cavity, where the $\mathrm{C}_{\alpha}-\mathrm{C}_{\beta}$ bond of the thioester substrate and the isoalloxazine ring of the FAD are located, is larger in the mutant than in the wild type enzyme, implying greater $\mathrm{O}_{2}$ accessibility in the mutant which might account for the higher oxygen reactivity.
\end{abstract}

Acyl-CoA dehydrogenases are a family of flavoenzymes that catalyze the $\alpha, \beta$-dehydrogenation of acyl-CoA thioesters to the corresponding trans-enoyl-CoA with transfer of reducing equivalents to electron transfer flavoprotein (Beinert, 1963). In mammalian mitochondria, four straight-chain specific acyl-CoA dehydrogenases [short (SCADH)-, medium (MCADH)-, long (LCADH)-, and very long chain (VLCAD) acyl-CoA dehydrogenase] involved in fatty acid catabolism (Beinert, 1963; Aoyama et al., 1994) and three branched-chain specific acyl-CoA dehydrogenases (isovaleryl-, 2-methylbutyryl-, and glutaryl-CoA-DH) in amino acid metabolism have been described (Tanaka \& Indo, 1992; Lenich \& Goodman, 1986). Among them, MCADH is the most studied: the three dimensional structure of pig MCADH has been determined with and without various substrates and

\footnotetext{
$\dagger$ This research was supported by grants from the National Institutes of Health, GM29076 and RR07178, awarded to J.-J.P.K. and by a grant from the Deutsche Forschungsgemeinschaft (Gh 2/4-7, Gh 2/6-1) to S.G.

* To whom correspondence should be addressed. Tel: (414) 4568479. E-mail: jjkim@ post.its.mcw.edu.

\# Medical College of Wisconsin.

$\S$ University of Konstanz.

${ }^{\otimes}$ Abstract published in Advance ACS Abstracts, September 1, 1996.

${ }^{1}$ Abbreviations: MCADH, medium-chain acyl-CoA dehydrogenase; MLCADH, medium long chain acyl-CoA dehydrogenase = Glu376Gly/ Thr255Glu mutant of MCADH; LCADH, long-chain acyl-CoA dehydrogenase; IVDH, isovaleryl-CoA dehydrogenase; CX-CoA, straightchain acyl-CoA thioester ligands, where $\mathrm{X}$ denotes the alkyl chain length.
}

inhibitors (Kim et al., 1993, 1994) and its catalytic residue has been identified (Powell \& Thorpe, 1988) and confirmed by site-specific mutagenesis (Bross, et al, 1990) and by the three dimensional structures of enzyme-substrate complex (Kim et al., 1993). All seven acyl-CoA dehydrogenases have been cloned, sequenced, and over-expressed in bacterial systems. Of the 27 known amino acid sequences of acylCoA dehydrogenases from both mammalian and bacterial sources available, the catalytic residue (Glu376, MCADH numbering) has been conserved except in LCADH and isovaleryl-CoA dehydrogenases (IVDH). The corresponding residue is a glycine in both LCADH and rat IVDH and is an alanine in human IVDH (Matsubara et al., 1990). On the basis of the crystal structure of pig MCADH, Djordjevic et al. (1994) have identified the catalytic residue of LCADH as Glu261 (255 in MCADH numbering) by molecular modeling and confirmed it by site-specific mutagenesis. Glu261 is topologically conserved in the three-dimensional structure but lies in a different segment of the polypeptide chain in the primary sequences (Figure 1). A similar study (Mohsen \& Vockley, 1995) confirmed that the catalytic base in IVDH is Glu254 (255 in MCADH numbering). In the preceding paper, a converse experiment has been performed in which both Glu376 and Thr255 of MCADH have been altered to the corresponding residues found in LCADH, glycine, and glutamate, respectively. The resulting double mutant (Glu376Gly/Thr255Glu) has its substrate specificity and turnover numbers similar to those of LCADH (Nandy et al., 1996, the preceding paper), thus making MCADH 


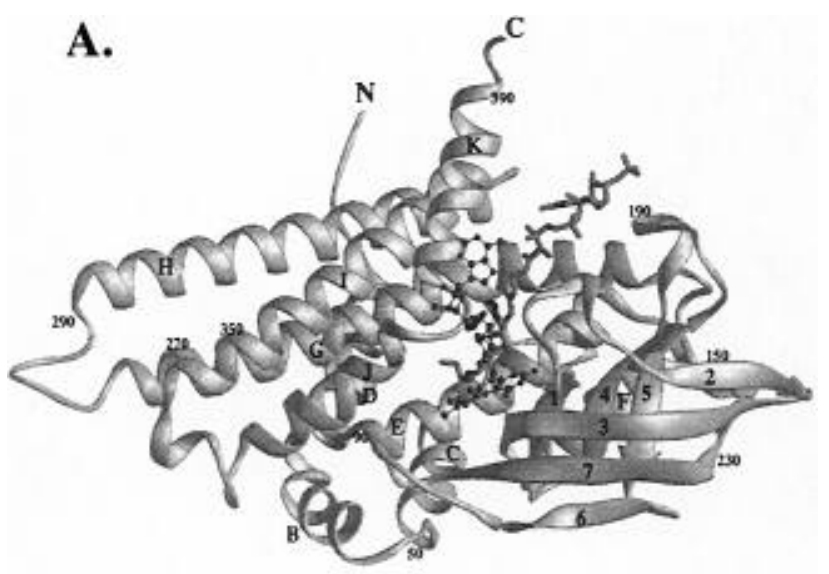

B.

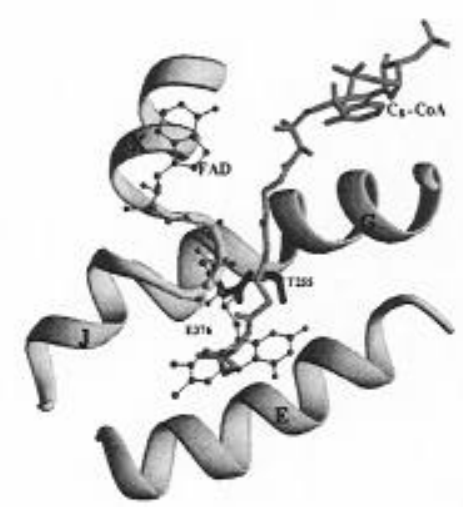

FIGURE 1: Ribbon diagrams of the overall structure of a monomer of MCADH (A) and an enlargement of the vicinity of E376 and T255 (B). $\alpha$-Helices are lettered and $\beta$-strands numbered consecutively from the N-terminus. The FAD is represented by ball-andstick, and octanoyl-CoA is represented by grey sticks. The side chains of E376 and T255 are represented by thicker black sticks.

specific for medium long chain acyl-CoA substrates (MLCADH). We have determined the three-dimensional structures of MLCADH with bound substrates having various fatty-acyl chain lengths. These structures illustrate the structural bases for the altered chain length specificity and for the dramatic differences in the oxygen reactivity between the wild type and the mutant. Furthermore, the detailed picture of the active site with bound ligand suggests a plausible mechanism of the $\alpha$-proton abstraction in MLCADH, which is most likely to be similar to the mechanism of LCADH and IVDH.

\section{MATERIALS AND METHODS}

Crystallization. The human wild type (MCADH) and the double mutant enzyme (Glu376Gly/Thr255Glu, MLCADH) were expressed in Escherichia coli and purified as described in the preceding paper (Nandy et al., 1996). The purified enzymes were stored as a slurry in $85 \%$ saturated ammonium sulfate solution at $-80{ }^{\circ} \mathrm{C}$. Immediately prior to the crystallization experiments, the enzyme was extensively dialyzed against $50 \mathrm{mM}$ potassium phosphate, $\mathrm{pH} 7.6$, and was reduced to a final concentration of $10 \mathrm{mg} / \mathrm{mL}$. Crystallization was carried out by the vapor diffusion method at 4 ${ }^{\circ} \mathrm{C}$ using the sitting drop technique (McPherson, 1985). $\mathrm{X}$-ray-quality crystals were obtained from crystallization drops $(5 \mu \mathrm{L})$, each containing $27 \mu \mathrm{g}$ of the enzyme in 140 $\mathrm{mM}$ Tris acetate, $\mathrm{pH} 7.0$, and $8.0 \%(\mathrm{w} / \mathrm{v})$ polyethylene glycol (PEG) 4000, that had been equilibrated against 50\% PEG 4000. The crystallization conditions for the enzymesubstrate complexes were similar to those for the uncom- plexed enzyme crystals except that the crystallization drops contained a 1.2 molar equivalent (per flavin) of the corresponding fatty acyl-CoA (C8-CoA, C12-CoA, or C14-CoA). In addition to the co-crystallization method, crystals of the complex with C14-CoA were obtained by a soaking method in which a solution of C14-CoA was added to the dips containing preformed crystals of the free enzyme. The crystallization setups for the complexes were performed in a glovebox purged with argon gas, and the crystallization plates were tightly sealed with vacuum grease and stored outside of the glovebox. However, no special precaution was taken to keep the crystals anaerobic during mounting of the crystals in glass capillaries for the diffraction data collection.

$X$-ray Data Collection. All data sets were collected using an R-AXIS IIC image-plate system with $\mathrm{Cu} \mathrm{K \alpha}$ radiation generated from a Rigaku RU-200 generator equipped with a graphite monochromator. The crystals mounted in a glass capillary were kept at $4{ }^{\circ} \mathrm{C}$ with a cold air stream during the data collection. Each data set was obtained from one crystal except for the set of the uncomplexed mutant enzyme which was from two crystals. The crystal to detector distance was $200 \mathrm{~mm}$; the $2 \theta$ angle for the detector, $10^{\circ}$; the oscillation angle, $0.8^{\circ}$; and the exposure time, $10 \mathrm{~min}$ per frame. The data sets were collected and reduced using the R-AXIS software package (Higashi, 1990). Crystals of both the wild type and the mutant were isomorphous. The space group was determined to be tetragonal, either $P 4_{3} 22_{1}$ or $P 4_{1} 2_{1} 2$, containing one tetrameric molecule per asymmetric unit. The unit cell dimensions and the data collection statistics are given in Table 1.

Structure Determination. The structure of the human wild type MCADH was solved by molecular replacement methods using the refined crystal structure of the pig MCADH (Kim et al., 1993) as the search model and the X-PLOR program package (Brunger, 1992). The sequence identity between pig and human MCADH is $90 \%$. A tetrameric model used in subsequent analysis was constructed by applying the crystallographic symmetry to the coordinates of the dimer structure of the pig enzyme. This model was used in a crossrotation search using the Patterson search procedure implemented in X-PLOR. Patterson correlation refinement of the peaks found in the rotation search gave four possible solutions (Table 2). Rotation values which gave the highest peak both in the rotation function and in the Patterson correlation was used for the subsequent translation searches. Since there was an ambiguity in the space group assignment, translation searches were carried out for the two possible space groups, $P 4_{3} 2_{1} 2$ and $P 4_{1} 2_{1} 2$. The resulting translation function solution clearly identified the space group to be $P 4_{3} 2_{1} 2$ (translation function value $=0.516, \sigma_{\mathrm{TF}}=0.013$, $R$-factor $=0.37$ for $P 4_{3} 2{ }_{1} 2$; translation function value $=$ $0.296, \sigma_{\mathrm{TF}}=0.012, R$-factor $=0.44$ for $\left.P 4_{1} 2_{1} 2\right)$. For both rotation and translation searches, 18044 reflections with intensities greater than $8 \sigma$ in the resolution range between 15 and $4 \AA$ were used. The structures of both uncomplexed and complexed crystals of MLCADH were determined by difference Fourier methods using phases obtained from the human wild type structure.

Refinement. All structures were refined using X-PLOR with manual adjustments between refinement cycles on a Silicon Graphics workstation using the Turbo graphics software (Cambillau \& Roussel, 1993). For the wild type enzyme, the solution obtained from the rotation - translation 
Table 1: Data Collection and Refinement Statistics for MCADH and MLCADH Crystals ${ }^{a}$

\begin{tabular}{|c|c|c|c|c|c|c|}
\hline & \multirow[b]{2}{*}{ MCADH } & \multicolumn{5}{|c|}{ MLCADH } \\
\hline & & & $\mathrm{C} 8-\mathrm{CoA}$ & $\mathrm{C} 12-\mathrm{CoA}$ & C14-CoA co-crystals & C14-CoA soaked \\
\hline resolution $(\AA)$ & 2.75 & 2.4 & 2.6 & 2.5 & 2.5 & 2.5 \\
\hline total no. of reflections ${ }^{b}$ & 104904 & 204212 & 137635 & 172577 & 133442 & 212299 \\
\hline no. of unique reflections & 44572 & 66928 & 51243 & 57376 & 55675 & 64950 \\
\hline$R_{\text {merge }}(\%)^{c}$ & 10.68 & 7.29 & 10.18 & 8.43 & 8.11 & 8.69 \\
\hline $\begin{array}{l}\text { completeness }(\%) \\
\text { unit cell }^{d}(\AA)\end{array}$ & 79.0 & 77.7 & 70.7 & 75.3 & 72.8 & 84.7 \\
\hline (a) & 169.59 & 170.18 & 170.15 & 170.00 & 170.24 & 170.35 \\
\hline (c) & 151.10 & 150.13 & 148.61 & 149.87 & 149.94 & 150.19 \\
\hline$R$-factor ${ }^{e}$ & 22.1 & 20.8 & 21.7 & 19.6 & 20.0 & 21.1 \\
\hline no. of water molecules & - & 230 & 139 & 252 & 215 & 191 \\
\hline $\begin{array}{l}\text { deviations in RMS geometry } \\
\text { bond }(\AA)\end{array}$ & 0.009 & 0.009 & 0.009 & 0.009 & 0.009 & 0.010 \\
\hline angles (deg) & 1.6 & 1.6 & 1.6 & 1.7 & 1.6 & 1.7 \\
\hline dihedrals (deg) & 22.3 & 22.5 & 22.6 & 22.3 & 22.6 & 22.9 \\
\hline improper (deg) & 1.3 & 1.2 & 1.5 & 1.3 & 1.3 & 1.3 \\
\hline
\end{tabular}

${ }^{a}$ All data sets were collected using one crystal per data set, except for the uncomplexed mutant, which was from two crystals. ${ }^{b}$ For all reflections, $I / \sigma(I)>1.0 .{ }^{c} R_{\text {merge }}=\sum_{h} \sum_{i=1, N}\left|I_{h i}-<I_{\mathrm{h}}>\right| / \sum_{h}\left(N<I_{\mathrm{h}}>\right)$, where $h$ are unique reflection indices and $i$ indicates symmetry equivalent indices. ${ }^{d}$ The space group for all crystals is $P 4_{3} 2{ }_{1} 2 .{ }^{e}$ Final $R$-factors were calculated with $10-2.5 \AA$ data.

Table 2: Rotation Searches of Human MCADH Using the Structure of Pig $\mathrm{MCADH}^{a}$

\begin{tabular}{|c|c|c|c|c|c|c|c|c|}
\hline \multirow{2}{*}{$\frac{\text { peak index }}{154}$} & \multicolumn{3}{|c|}{ before $\mathrm{PC}$ refinement } & \multicolumn{3}{|c|}{ after $\mathrm{PC}$ refinement } & \multirow{2}{*}{$\frac{\text { rotation function }}{0.9687}$} & \multirow{2}{*}{$\frac{\text { Patterson correlation }}{0.1108}$} \\
\hline & 140.53 & 42.0 & 65.18 & 140.40 & 41.9 & 65.31 & & \\
\hline 985 & 137.46 & 48.0 & 83.64 & 139.68 & 41.3 & 88.27 & 0.9224 & 0.1101 \\
\hline 3604 & 221.03 & 48.0 & 200.62 & 220.12 & 42.4 & 204.00 & 0.8678 & 0.1101 \\
\hline 4952 & 218.68 & 42.0 & 183.10 & 220.76 & 41.7 & 181.04 & 0.8510 & 0.1100 \\
\hline
\end{tabular}

${ }^{a}$ The space group of pig MCADH is $C 222_{1}$, and the cell dimensions are $a=128.82, b=136.14$, and $c=106.13 \AA$. The asymmetric unit contains two monomers of the tetrameric molecule.

searches were subjected to the rigid body refinement followed by Powell positional refinement. At this stage, a $2 F_{\mathrm{o}}$ $-F_{\mathrm{c}}$ map was calculated, and the side chains of the residues that are not conserved between the pig and human MCADHs were corrected to the corresponding residues of the human enzyme when the densities fit, otherwise changed to alanine. Each subsequent cycle of refinement included positional refinement followed by simulated annealing with a slowcool protocol from 2000 to $300{ }^{\circ} \mathrm{C}$. After one round of the simulated annealing refinement, all side chains were fitted with those of the human enzyme. All four monomers in the asymmetric unit were restrained with non-crystallographic symmetry until the R-factor dropped between $23 \%$ and $24 \%$. At this point, all four monomers were treated independently, and at the final cycle of refinement individual temperature factors were used. Refinements for the uncomplexed MLCADH structure were started with the final coordinates of the wild type structure. A rigid body refinement was carried out first, followed by the similar refinement protocol used for the wild type structure. A difference Fourier map confirmed the mutation, and the side chains of the two altered residues (Glu255 and Gly376) were modeled. The refinements of the structures of the complexes were carried out in the same manner, except that the starting model was the refined structure of the uncomplexed mutant enzyme. The bound ligand molecules in the complex structures were modeled from difference Fourier maps in the beginning stages of the refinements. A typical $2 F_{\mathrm{o}}-F_{\mathrm{c}}$ map of MLCADH/C8-CoA showing the densities of the bound ligand, C8-CoA, is shown in Figure 2. In the case of MLCADH/C12-CoA, two different orientations of the $\omega$-end of the bound ligand was conformed by omit maps and also by refining the structure in both conformations and examining the difference Fourier maps. At the later stages of the

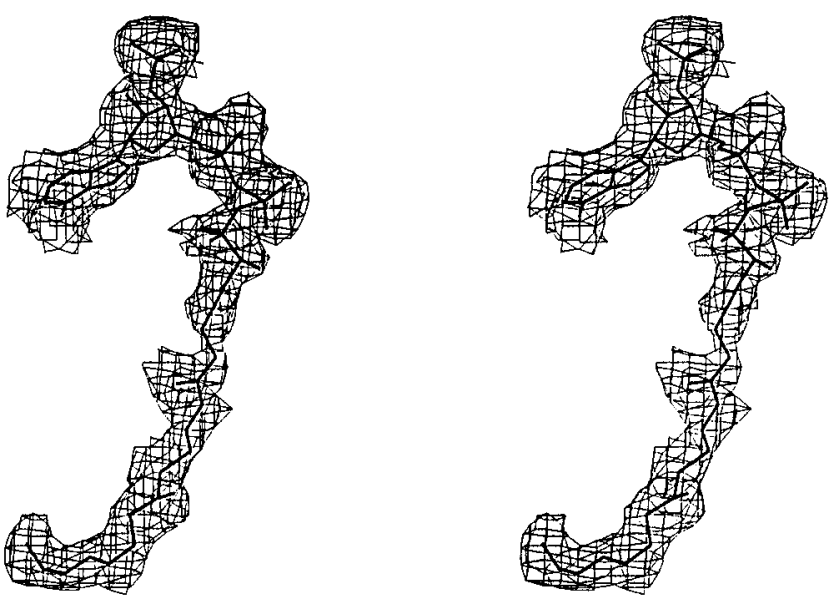

FIGURE 2: Stereodiagram of a model of C8-CoA fitted in the final $2 F_{\mathrm{o}}-F_{\mathrm{c}}$ map of MLCADH complexed with C8-CoA. The electron density was contoured at the $1.2 \sigma$ level.

refinements, water molecules were added when densities were higher than $3 \sigma$ in difference fourier maps $\left(F_{\mathrm{o}}-F_{\mathrm{c}}\right.$ map) and in positions acceptable for forming hydrogen bonds with either protein atoms or other water molecules. Because of the relatively lower resolution, no water molecules were added to the wild type structure. Table 1 summarizes the results of data collection and the structure refinements.

Solvent Accessible Area. Solvent accessible areas of the molecules were calculated and displayed using the Connolly surface (Connolly, 1983) protocol incorporated in the TURBO package. The probe radius used was $1.6 \AA$.

\section{RESULTS}

Structures of Both Pig and Human MCADHs Are the Same. Amino acid sequence comparison between the human 

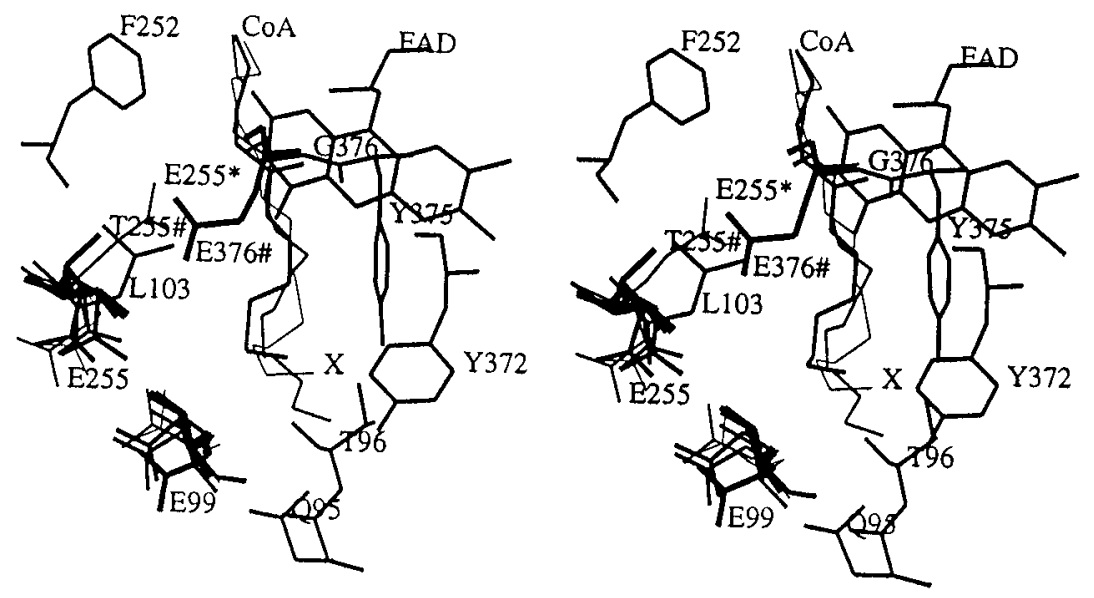

FIGURE 3: Stereodiagram of a superposition of the active site in MLCADH complexed with C8-, C12-, and C14-CoA. For comparison, E376 and T255 of the structure of pig MCADH complexed with C8-CoA are drawn in with thickest lines and marked with "\#". For the mutant structures, C8-CoA is shown in thick lines, C12-CoA in thinner lines, and C14-CoA in thinnest lines. Since the tail-end of the C14-CoA is disordered, the alkyl chain of the C14-CoA is shown up to the C10 atom, which is marked with an "X". For clarity, only the side chains of E255 and E99 of all three complexes are shown. The other residues in the active site and the FAD shown are from the structure of C8-CoA complex, since their conformations are the same in all complexes. Two conformations of E255 are shown, and E255* denotes the "active" conformer.

and the pig enzyme shows that they are $90 \%$ identical. Of the total 386 residues (excluding the first 10 residues from the amino terminus that are not well defined in the crystal structures), 28 are conservative replacements and only 13 residues are different, none of which is located in the active site. More importantly, the root-mean-square (rms) deviation of the main chain atoms between the two wild type structures is $0.27 \AA$ and those between two monomers in the pig and among four monomers in the human structures are 0.21 and $0.27 \AA$, respectively. Therefore, both structures are essentially the same. Since the structure of human MCADH complexed with substrate has not been determined, we use in this report the structure of the pig MCADH complexed with octanoyl-CoA for comparison with the structures of the mutant complexes.

Nature of the Bound Ligands and the Oxidation State of the Flavin. The initial crystallization solutions for the complexes of the mutant with $\mathrm{C} 8-\mathrm{CoA}$ and $\mathrm{C} 12-\mathrm{CoA}$ were dark brown in color, indicating that the flavin in the enzyme was at least partially reduced, the substrate was oxidized, and the charge transfer complex was formed. However, the solution turned to the bright yellow within a relatively short time (within hours for $\mathrm{C} 12-\mathrm{CoA}$ to overnight for $\mathrm{C} 8-\mathrm{CoA}$ ) even in a glovebox, and the fully grown crystals of all complexes ( $2 \sim 4$ weeks until ready for data collection) appeared to have the bright yellow color, suggesting that the flavin was completely reoxidized. In the case of the C14CoA complex, even the initial color change upon addition of C14-CoA was not apparent, presumably due to the rapid reoxidation of the substrate-reduced enzyme by the molecular oxygen dissolved in the solutions. Therefore, no additional precautions were taken to keep the crystals anaerobic while mounting them for the X-ray data collection. Since the initial amount of the substrate was a slight excess of the flavin, the bound ligand in the crystals must be a mixture of the substrate and the product in all cases. Although the exact ratio of substrate:product could not be determined, the predominant form of the bound ligand in all complexes was probably the product (see Table 1 of the preceding paper). Therefore, we modeled the bound ligands as products (trans2,3-enoyl-CoA), and refer to them as C8-, C12-, or C14$\mathrm{CoA}$, indicating their chain length only.
Comparison among the Structures of the Mutant Complexes. The rms deviations for the main chain atoms among different structures including the wild type and those among different monomers within each tetramer structure range from 0.2 to $0.3 \AA$, indicating that there is no significant difference in the overall protein folding and in the quaternary structure between the wild type and the mutant enzyme and among the structures of different complexes. However, there are a few subtle but significant differences in side chain conformations among various structures, which will be discussed below. Unless specifically mentioned, the structure of the complex with $\mathrm{C} 8-\mathrm{CoA}$ is used as the representative of the complexed structures. Figure 3 shows a superposition of the residues in the binding pocket of the fatty acyl moiety found in the structures of wild type and the mutant complexes with C8-CoA, C12-CoA, and C14-CoA. The conformation of Glu99 is different between the wild type and the mutant structures, and those of Glu255 and Glu99 are different among the complexes depending on the location of the fatty acyl "tail-end" in the active site cavity. In the mutant, Glu255 and Glu99 appear to form a hydrogen bond at the base of the alkyl chain binding cavity. It is not unusual to observe such carboxyl-carboxylate hydrogen bonding interactions in which the two carboxyl groups share a proton (Sawyer \& James, 1982). It is likely that the $\mathrm{p} K_{\mathrm{a}}$ of Glu99 in the mutant is perturbed to a higher value than that of the wild type enzyme. In the wild type, the corresponding residue at the position 255 is a threonine and does not form a hydrogen bond with Glu99 (Figure 3). There are no other significant differences between the uncomplexed and the C8CoA complexed structures of the mutant, therefore for clarity, the uncomplexed mutant structure was not included in the figure. In the case of the pig liver enzyme, although the overall- and the main chain structures of the uncomplexed and the complexed enzyme are the same, a few but distinctive differences have been observed in the side chain conformations of the residues at the active site, such as those of Tyr375, Glu99, and Gln95 (Kim et al., 1993). However, in the case of the mutant, no significant differences in the side chain conformations between the uncomplexed and the complexed structures were observed. Whether this is due to the relatively low resolution at which the mutant structures 


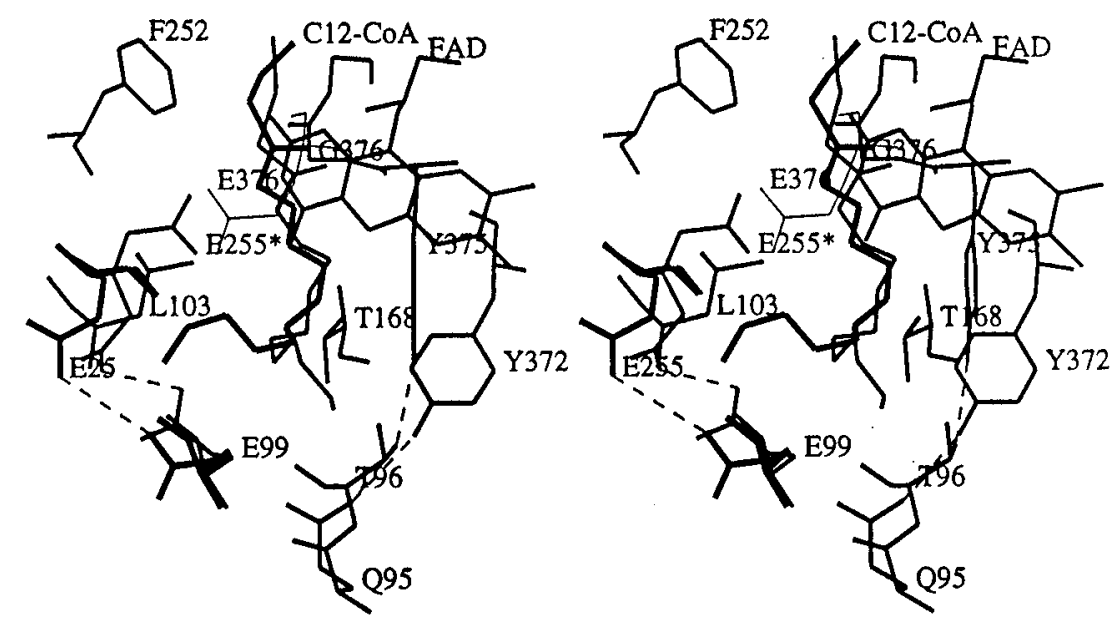

FIGURE 4: Superposition of the active sites of the two representative monomers of MLCADH complexed with C12-CoA whose $\omega$-end binds to the enzyme in two different directions. The end of one monomer points to the direction of the hydrogen-bonding network formed by Y372, Y375, Q95, and T96 (Monomer 1, the predominant form). The minor form (observed in monomer 2) points to the direction of the hydrogen bond between E255 (the "inactive" conformer) and E99. Neither monomer 3, whose conformation is the same as that of monomer 1, nor monomer 4, in which the tail-end is disordered, is shown for clarity. Dashed lines represent potential hydrogen bonds. Both conformers of E255 are shown and the "active" form is denoted as E255*. For comparison, wild type E376 is shown and marked with “\#”.

were determined, or whether the two structures (complexed and uncomplexed) are identical, awaits further studies.

The $\omega$-End of C12-CoA Binds to the "Base" of the Active Site Cavity in Two Different Directions, and That of C14CoA Is Disordered. Electron densities in the difference Fourier maps of both C8- and C12-CoA could be fitted easily with the molecular models having their respective length of the alkyl chains. Figure 2 depicts a model of $\mathrm{C} 8-\mathrm{CoA}$ in the final $2 F_{\mathrm{o}}-F_{\mathrm{c}}$ difference electron density map contoured at $1.2 \sigma$ level. In all of the enzyme crystals, there is one tetrameric molecule per asymmetric unit. The conformation of all four enzyme-bound C8-CoA molecules in the tetramer of the C8-CoA complex are essentially the same within the experimental error. On the other hand, the densities for the $\omega$-end of the C12-CoA could be fitted in two different conformations, two monomers showed predominantly in one and the third monomer in both directions with approximately equal proportion. The occupancy of the bound ligand in the fourth monemer is relatively low, and its $\omega$-end is not well defined. The predominant direction of the alkyl tail-end is the same as that observed in the crystal structure of the pig MCAD complexed with C12-CoA (Kim et al., 1994). A superposition of the active sites of the two representative monomers in the structure of the C12- CoA complex (Figure 4) shows that the "tail-end" (C10 through $\mathrm{C} 12$ atoms) of the enzyme-bound ligand extends in two different directions with an overall ratio of the two conformers of approximately 3:1. Since the tail-end of the alkyl chain is located in the interior of the protein, the conformation of the alkyl chain should not be influenced by crystal packing. Therefore the significance of the different ratios between two conformers found in different monomers is not clear at present. Furthermore, the difference Fourier maps of the C14-CoA complex could be fitted with only either C9- or C10-CoA. Since the alkyl chain of the C14-CoA could not have been cleaved during the normal crystallization procedure, the $\omega$-end (C11 through C14) of the C14-CoA must be disordered in the crystal structure. In an attempt to locate the $\omega$-end of the bound C14-CoA, we have solved the structure of another crystal of C14-CoA complex that had been prepared by soaking C14-CoA into a preformed uncomplexed enzyme crystal. The resulting structure was the same as that

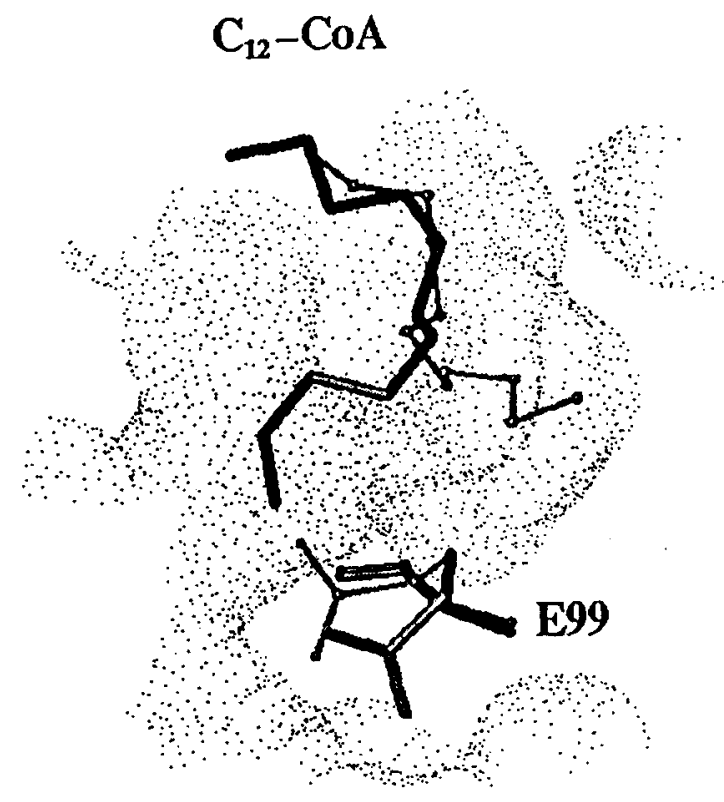

FIGURE 5: Solvent accessible area at the "base" of the active site cavity in the structure of MLCADH complexed with C12-CoA. Only the alkyl chain portion of the bound C12-CoA and the side chain of E99 are shown. The view is approximately the same as the one shown in Figure 4. Monomer 1 is represented by thin lines and monomer 2 in thick lines. With a minor movement of the side chain of E99, the alkyl chain can be accommodated in the active site cavity in two different conformations. The radius of the probe used to generate the solvent accessible area was $1.6 \AA$.

of the co- crystal within the error limits of both structures including the disordered feature of the $\omega$-end of the bound C14-CoA. The rms difference between the main chain atoms of the soaked and co- crystal structures is $0.25 \AA$. Thus, it is reasonable to assume that the ligand with fatty-acyl chain length longer than $\mathrm{C} 10$ is flexible, and that its $\omega$-end binds to the enzyme in multiple conformations. The solvent accessible area at the "bottom" of the active site cavity found in the C12-CoA complex is shown in Figure 5. With a minor movement of the side chain of Glu99 together with its hydrogen bonding partner, Glu255, the C12-CoA molecules can be accommodated in two different directions at the base of the binding cavity. However, there appears to be no apparent correlation between the two distinct conformations 

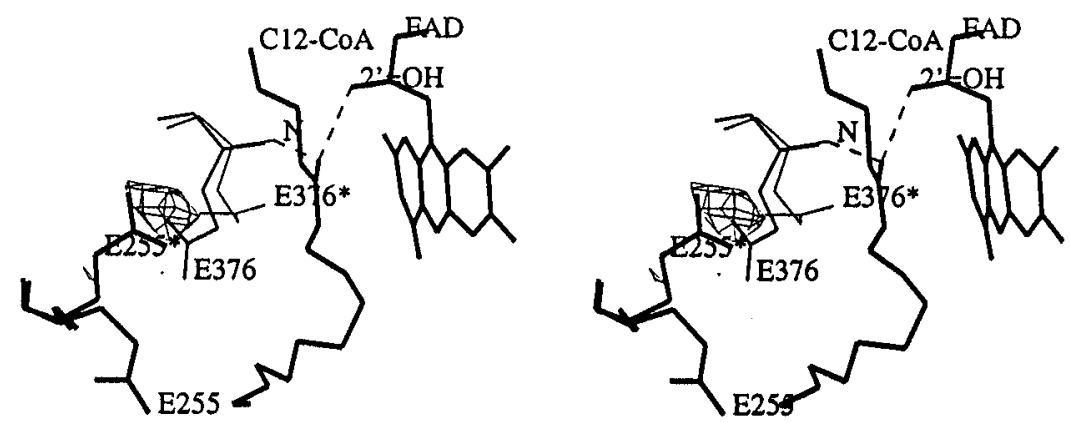

FIGURE 6: Two conformers of E255 found in the structure of the complex between MLCADH and C12-CoA. The final $2 F_{\mathrm{o}}-F_{\mathrm{c}}$ map using the structure of the "resting" conformer of E255 shows a ball of density that cannot be accounted for by a water molecule because there is no protein atom with which a hydrogen bond can be formed. The density is contoured at the $0.8 \sigma$ level and can be fitted with a model of the carboxylate of E255, the "active" conformer (denoted as E255*). For comparison, the two conformations of E376 found in pig MCADH are shown in thinner lines, E376* of the uncomplexed MCADH and E376 of the structure of MCADH complexed with C8-CoA. Presumably the true active conformer of E376 in MCADH is similar to that found in the uncomplexed form (E376*).

of Glu255 (see below) and the two different conformations of the tail-end of the C12-CoA.

In the Mutant Structure, Glu255 Is the Catalytic Residue and It Has Two Conformations. The conformation of the Glu255 side chain found in the initial difference Fourier map is located over $7 \AA$ (6.9-8.6 $\AA$ depending on the structures) away from the $\mathrm{C}_{\alpha}$ atom of the bound ligand. This suggests that this conformer is not an active form of the enzyme (hereafter, referred as the "resting" form). However, the final $F_{\mathrm{o}}-F_{\mathrm{c}}$ maps for the structures of the complexes of C12and C14-CoA showed a strong difference electron density near the $\mathrm{C}_{\alpha}$ atom (about $4.5 \AA$ away) which could not be accounted for by a solvent molecule, since there was no protein atom with which a hydrogen bond could be formed. Figure 6 shows a density observed in the final $2 F_{\mathrm{o}}-F_{\mathrm{c}}$ map of the C12-CoA complex that could be fitted with the carboxylate of Glu255. In this conformation, the Glu255 carboxylate is situated at the opposite side of the $\mathrm{C}_{\alpha}-\mathrm{C}_{\beta}$ bond from the isoalloxazine ring capable of $\alpha$-proton abstraction ("active" form). In an attempt to estimate the ratio of the "active" form and the "resting" form, the occupancies and the positions of the two alternate conformations were used in the structural refinement. The resulting ratios of the occupancies between the "active" and "resting" conformers ranged from 0:1.0 to 0.3:0.7 depending on the monomers of the tetrameric molecule. On average, approximately $10 \%-15 \%$ of the total enzyme population exists in the "active" conformation in both the C12- and C14-CoA complexes. No corresponding densities attributable to the "active" conformer were observed in the structures of the uncomplexed or C8-CoA-bound forms of the enzyme. It is not clear whether there is any relationship between the percentage of the active conformer observed in the crystalline state and the catalytic activity of MLCADH toward the substrate with which the enzyme was crystallized. Also not clear is whether there is any correlation between the presence of the "active" conformer and the multiple conformers of the $\omega$-end of the enzyme-bound ligand. Further experiments are required to clarify this point. In the "resting" state, the Glu255 carboxylate is stabilized by forming a hydrogen bond with Glu99 at the base of the active site cavity whereas no such interactions are observed stabilizing the "active" conformer (Figure 4).

The Mutant Structure Has More Room at the Midsection of the Active Site Cavity than the Wild Type. The ligand binding site forms a long tubular cavity (about $20 \AA$ deep) with a funnel-shaped opening. The fatty-acyl group of the thioester ligand occupies the base of the cavity and is deeply buried in the interior of the protein, whereas the adenosine moiety is located at the opening of the cavity and lies at the surface of the enzyme molecule (Kim et al., 1993; also shown in Figure 1). As in the wild type enzyme, the thioester carbonyl oxygen is situated at the midsection of the long cavity and within hydrogen bonding distance from both 2'$\mathrm{OH}$ of the FAD ribityl group and the amide nitrogen of Gly376. In the wild type structure, the midsection of the cavity, where the carbonyl oxygen of the thioester and the $\mathrm{C}_{\alpha}-\mathrm{C}_{\beta}$ bond are located, is formed by the flavin ring, Glu376, Tyr375, Phe252, and Leu103, and is very constricted. In the mutant structures, on the other hand, the [-carbonyl group- $\mathrm{C}_{\alpha}-\mathrm{C}_{\beta^{-}}$] portion of the ligand fits in the active site cavity relatively loosely. Solvent accessible areas at the active site in both the wild type and mutant structures were calculated using the structures of the complexes with C8-CoA but excluding the bound ligand (Figure 7). The diameter of the cross-section of the tubular cavity at the $\mathrm{C}_{\alpha}-$ $\mathrm{C}_{\beta}$ position is about $5 \AA$ in the wild type, whereas that of the mutant is about $8 \AA$. The same results were obtained for C12-CoA complexes (data not shown) as for the C8CoA complexes, in which the fatty acyl binding cavity is also wider in the mutant than in the wild type enzyme. Thus the active site in the mutant is more solvent, and presumably more oxygen, accessible than that of the wild type enzyme. In the pig MCADH structures (Kim et al., 1994), the active site cavity in the uncomplexed enzyme is occupied by a string of water molecules and these water molecules are displaced by the bound substrates in the structures of the complexes. The number of water molecules displaced is dependent on the substrate chain length, and when C10-CoA binds, all the water molecules in the cavity are displaced. In the uncomplexed MLCADH structures, only one water molecule was found in the active site cavity at the location where the carbonyl oxygen of the substrate occupies in the structure of the complex. Water molecules in the cavity are presumably disordered due to the larger space of the cavity.

\section{DISCUSSION}

Many earlier studies of MCADH, including detailed biochemical, spectroscopic, and kinetic studies, were obtained from the pig enzyme. However, the recent focus has been on the human enzyme due to the recognition of MCADH deficiencies as the most common disorders of fatty acid oxidation in human (Gregersen et al.,1993). Our current work confirms that the three dimensional structures of the 

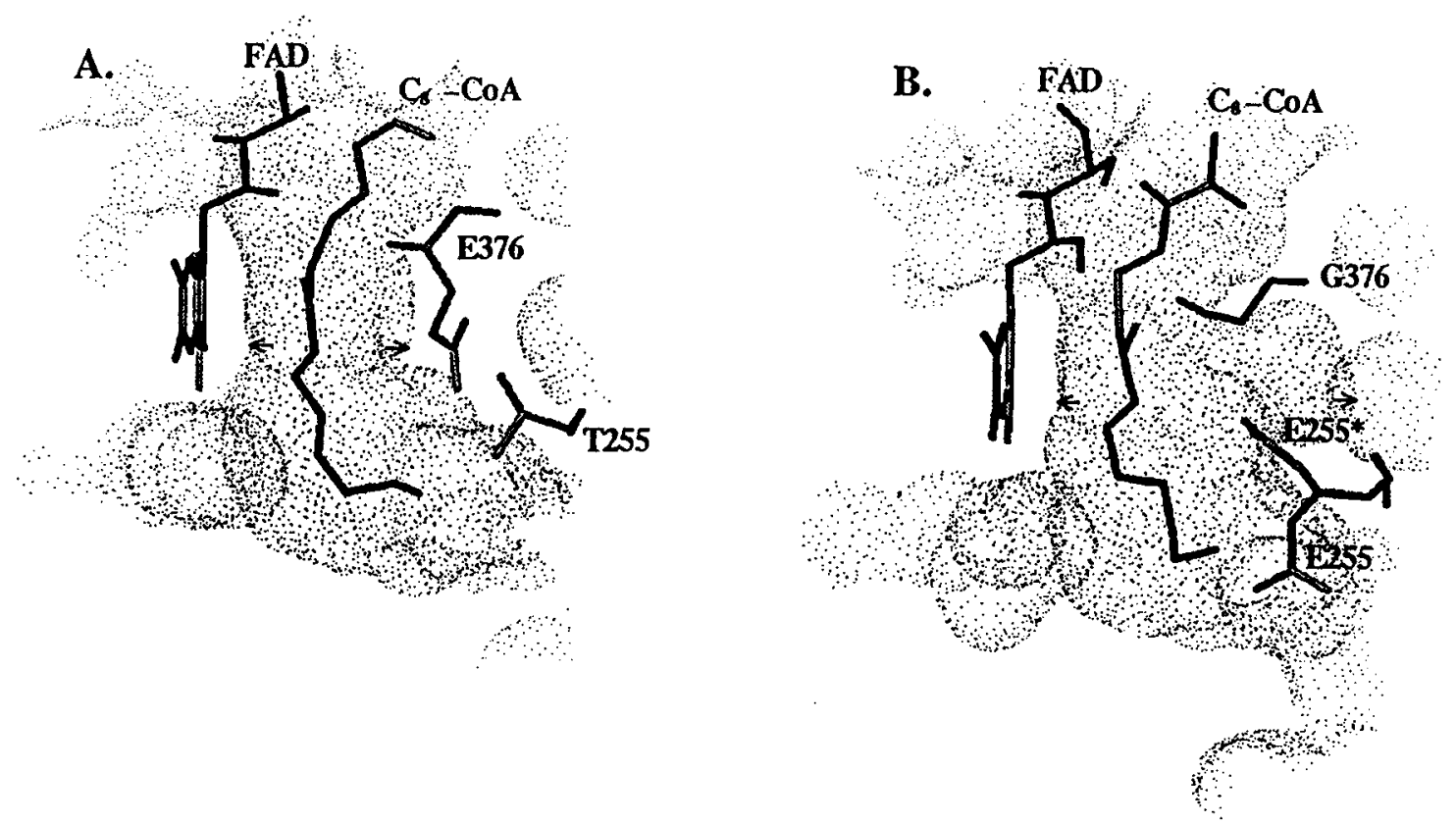

FIGURE 7: Comparison of the solvent accessible areas at the active site cavity found in the structures of complexes of pig MCADH (A) and of human MLCADH (B) with C8-CoA. Both conformers of E255 of MLCADH are shown in B. The diameters of the cross-section at the midsection (shown with arrows) of the cavity are approximately $5 \AA$ in MCADH and $8 \AA$ in MLCADH, suggesting that the active site of the mutant is more solvent accessible than that of the wild type.

pig and the human MCADHs are the same. Therefore conclusions drawn from the studies on the pig enzyme can be applied to the human enzyme.

The two conformations of Glu255 and the role of Glu99 in MLCADH. Glu255 exists in two conformations, "active" and "resting", and their distribution in the crystalline state is approximately 0.1:0.9 in favor of the "resting" form, presumably due to the stabilization obtained by forming a hydrogen bond with Glu99. Since both glutamates lie in the interior of the enzyme molecule, the conformation of Glu255 should not be influenced by the crystal packing force. Consequently, it is reasonable to assume that the equilibrium ratio of the two conformers in solution is approximately the same as in the crystalline state, i.e., "active"/"resting" conformers $=\sim 0.1$. It should be noted that MCADH is the only one that has a glutamate at position 99 among acylCoA dehydrogenases whose amino acid sequences are known. LCADH and IVDH have serine and glycine, respectively, at the corresponding position. Since neither the serine (too short) nor the glycine (no side chain) would be able to form a direct hydrogen bond with the "resting" conformer of Glu255, the corresponding glutamates in LCADH and IVDH are more likely to exist in their active conformers which, in turn, should contribute to the higher catalytic activity than that of MLCADH with their respective optimal substrate. It would be intriguing to see whether the mutant Glu99Ser of MLCADH (i.e., a triple mutant of MCADH, Glu99Ser/Glu376Gly/Thr255Glu), would be more active than MLCADH.

Comparison between Glu376 and Glu255 in Their Catalytic Abilities. There are at least two factors to be considered that would affect the catalytic ability of the carboxylate base. The first one is the distance between the proton to be abstracted (the $\mathrm{C}_{\alpha}$ pro- $R$ proton) and the base that abstracts the proton. The distances between the $\mathrm{C}_{\alpha}$ atom and the carboxylate oxygens of Glu376 in the wild type and those of Glu255 in the mutant are greater than $4.0 \AA$ A. However, it should be noted that the conformation of Glu376 observed in the structure of the wild type enzyme complexed with C8-CoA (Kim et al, 1993; also Figure 7) is that of the oxidized enzyme-product complex. For the optimal catalysis, Glu376 would most likely adapt a conformation similar to the one observed in the uncomplexed wild type structure (E376* in Figure 6), in which the carboxylate oxygen lies as close as $2.4 \AA$ from the $\mathrm{C}_{\alpha}$ atom. This distance is similar to that observed $(2.8 \AA)$ between the Glu165 carboxylate oxygens and the nitrogen atom of phosphoglycolohydroxamate (an inhibitor) found in the structure of triose phosphate isomerase (TIM) complexed with the inhibitor (JosephMcCarthy et al., 1994). Glu165 of TIM is the catalytic base that abstracts the proton from the substrate, dihydroxyacetone phosphate. On the other hand, the carboxylate oxygen atoms of the "active" conformer of Glu255 (E255* in Figure 2, 4, and 6) in the mutant structures are positioned over $4.0 \AA$ away from the $\mathrm{C}_{\alpha}$ atom of the substrate, and this is the closest approach for any conformation of the glutamate without altering the position of the main chain atoms. Therefore, the less efficient reduction rate of Glu255 than that of Glu376 is at least partly due to the longer distance between the carboxylate ion and the proton to be abstracted. For MLCAD, the reduction rate $\left(k_{\text {red }}\right.$, see Table 1 of the preceding paper) is reduced to 0.5 to $10 \%$ of the wild type depending on the substrate chain length. In the case of TIM, the catalytic rate $\left(k_{\mathrm{cat}}\right)$ of the Glu165Asp mutant, in which the catalytic base is separated from the substrate by about $1 \AA$, is reduced to $0.1 \%$ of the wild type. This suggests that in MLCADH the main chain flexibility at Gly376 might allow the carboxylate of Glu255 to be closer to the $\mathrm{C}_{\alpha}$ atom during catalysis. Alternatively and more likely, other factors are influencing the reduction of the $k_{\text {cat }}$ in the case of Glu165Asp mutant of TIM (see below). The above interpretation is consistent with the results of the mutant studies of MCADH and of IVDH in which their respective catalytic base was replaced with aspartate. The Glu376Asp mutant of MCADH has considerable activity (less than $10 \%$ of the wild type) by the ferricenium assay method (Ghisla et al, 1992), whereas 
the homologous mutant of IVDH, Glu254Asp, shows a barely detectable (less than $0.1 \%$ of the wild type) activity (Mohsen \& Vockley, 1995). IVDH has an alanine at amino acid position 375 (corresponding position of MCADH 376) which is more rigid than a glycine. The second factor that effects the general base catalysis by the carboxylate is the orientation of the carboxylate with respect to the proton to be abstracted. Examination of structure/mechanism studies of several enzymes that employ carboxylate catalysis, a syn orientation is favored over an anti orientation by a factor of between 10 and 100 (Gandour, 1981). Also, Asp165 of the TIM mutant has an anti orientation which probably is largely responsible for the extremely low catalytic activity compared to the syn carboxylate of Glu165 in the wild type (JosephMcCarthy et al., 1994). Even though the exact orientations of the carboxylates of Glu255 in MLCADH and of Glu376 in MCADH cannot be determined, their relative orientations with respect to the $\mathrm{C}_{\alpha}$ atom of the bound ligand can be estimated. The angle formed by $\mathrm{C}_{\gamma}$ atom of Glu376, $\mathrm{C}_{\alpha}$ of substrate, and $\mathrm{C}_{\gamma}$ of Glu255, with the $\mathrm{C}_{\alpha}$ atom at the vertex is about $40^{\circ}$. This implies that the direction in which Glu255 abstracts the proton is about $40^{\circ}$ away from that of Glu376, assuming that the substrate binds to the enzyme approximately in the same orientation in the wild type as in the mutant enzyme. Since the carboxylate of the thioester substrate forms hydrogen bonds with the $2^{\prime}-\mathrm{OH}$ of the FAD on one side and the amide nitrogen of the main chain of 376 on the other side, the orientations of the $\mathrm{C}_{\alpha}-\mathrm{C}_{\beta}$ bond of the bound substrate in both MCADH and MLCADH have to be the same within the flexibility of the main chain. Both of these two factors, i.e., the distance and the orientation of the carboxylate with respect to the $\mathrm{C}_{\alpha}$ proton, attribute to the intrinsic smaller $k_{\text {red }}$ of Glu255 compared to that of Glu376 for their respective optimum substrates. The $k_{\text {red }}$ for MLCADH is as small as 2 orders of magnitude less than that of MCADH (Nandy et al., 1996). However, it is not apparent from the structures why, in the wild type enzyme, there is an order of magnitude drop in the $k_{\text {red }}$ values between C10- and C12-CoA and also between C12- and C14-CoA [for detail, see Table 1 in the preceding paper by Nandy et al. (1996)]. It is conceivable that the orientation of the $C_{\alpha}$ proton might be changed slightly in order to accommodate the longer substrates in the binding cavity, at the expense of losing the optimum configuration/orientation of the carboxylate with respect to the $\mathrm{C}_{\alpha}$ proton. For MLCADH, in contrast, the orientation factor might not be as critical as in the wild type. The "loose" fit of the substrate at the active site in the binding cavity of MLCADH (Figure 7) allows more room to "twist" the alkyl chain to align the $\mathrm{C}_{\alpha}$ proton with the carboxylate of Glu255 for optimum orientation. In addition, the glycine at position 376 in MLCADH, whose amide nitrogen is hydrogen bonded to the carbonyl oxygen of the thioester, is more flexible than Glu376 of MCADH to allow the "twist" of the $\mathrm{C}_{\alpha}-\mathrm{C}_{\beta}$ bond for optimum orientation toward the carboxylate. This is in agreement with the observation that the variations in $k_{\text {red }}$ for the mutant with substrate chain length is only about 3 -fold between $\mathrm{C} 8$ - and C10- or C12-, and between C12- and C14-CoA, with a maximum rate with C10-CoA (Nandy et al., 1996). Another distinction between the action of Glu376 and of Glu255 lies in their interactions with 2-octynoyl-CoA. MCADH is irreversibly inhibited by 2-octynoyl-CoA by covalent modification at Glu376 (Powell \& Thorpe, 1988), but neither beef liver LCADH (Ankele et al., 1991) nor MLCADH (Nandy et al., 1996) is covalently modified by the inhibitor. Although 2-octynoyl-CoA is shown to be a mechanism-based inhibitor involving rate-limiting removal of one of the $\mathrm{C}_{\gamma}$ protons (Lau et al., 1988), the exact site of the covalent linkage with the glutamate on the inhibitor is not known at present. Even though the exact mechanism of the covalent bond formation between the inhibitor and the catalytic base is not clear, it is still consistent with the fact that Glu255 is farther away from, and is less properly oriented with, either the $\mathrm{C}_{\alpha}$ or $\mathrm{C}_{\gamma}$ atom to be able to directly interact with the inhibitor for the covalent modification than Glu376 is (Figure 6).

When Both Glutamates Are Available. As evident in Figure 6, when both Glu376 and Glu255 are present in the Thr255Glu mutant (Glu/Glu mutant), Glu376 can act as the catalytic residue and Glu255 adopts the "resting" conformation. This interpretation is in agreement with the kinetic parameters of this mutant with its optimum substrates $(\mathrm{C} 8$ and $\mathrm{C} 10-\mathrm{CoA})$ that are similar to those of the wild type enzyme (Nandy et al., 1996). Both in the wild type and MLCADH, the substrates with the alkyl chain length longer than $\mathrm{C} 12-\mathrm{CoA}$ can be accommodated, by forming multiple conformers at its $\omega$-end in the active site cavity. However, in the Glu/Glu mutant, the steric hindrance caused by the side chains of both glutamates prevents the binding of the longer substrate in an orientation for the catalysis to occur.

Solvent Accessibility and Oxygen Reactivity. As depicted in Figure 7, the wider bore of the active site cavity at its midsection in the MLCADH compared to the wild type enzyme (8.5 $\AA$ vs $5.1 \AA$ ) allows the longer substrate to be accommodated for catalysis by adopting multiple conformations at its $\omega$-end. It also permits bulkier substrates such as 4-nitrophenyl-acetyl-CoA to bind the enzyme more easily. Furthermore, this "extra space" in the active site of MLCADH allows more solvent molecules, which in turn allows more molecular oxygen dissolved in the solvent, accessible to the reduced flavin. Thus, this solvent accessibility in MLCADH makes the reoxidation of the reduced flavin about 10 to 100 times faster than the wild type. It is a wellestablished fact that reduced MCADH with bound product (or product analogs) is reoxidized by molecular oxygen much slower than the reduced enzyme without the bound ligand (Beinert, 1963; Wang \& Thorpe, 1991; Trieval et al., 1995). In their studies with redox-inactive analogs, Thorpe and coworkers (Trievel et al., 1995) have shown that the ligands which lack the carbonyl oxygen (e.g., thioether) are much less effective (at least 2 orders of magnitude) in protecting the reduced enzyme flavin toward molecular oxygen. This could be explained as following: First, the thioether ligand would bind less tightly to the enzyme than the corresponding thioester, due to lack of the two hydrogen bonds between the carbonyl oxygen to the flavin ribityl $2^{\prime}-\mathrm{OH}$ and to the main chain amide nitrogen of amino acid 376. This allows product dissociation more readily, which in turn increases solvent accessibility to the active site. Second, there would be more solvent accessible area at the active site even when the ligand is bound to the enzyme if it does not have the thioester carbonyl group. The "extra space" in the active site cavity is generated by the protein in the case of MLCADH, whereas it is from the lack of the carbonyl oxygen of the bound ligand in the thioether complex. On the other hand, the dramatic increase in the oxygen reactivity with substrate longer than C10-CoA observed in both the wild type and MLCADH can be directly correlated with the 
product dissociation (Srivastava et al., 1995; Nandy et al., 1996). Although it is not possible to determine the exact occupancies of the bound ligand in the crystal structures of each complex due to the relatively lower resolution, it is evident, from the levels of electron densities in the final $2 F_{\mathrm{o}}$ $-F_{\mathrm{c}}$ maps and the refined temperature factors, that the occupancies of the bound ligands in the C12-and C14-CoA complexes are much lower than that of the $\mathrm{C} 8-\mathrm{CoA}$ complex, again implying that the longer product (C12- and C14-CoA) binds less tightly to (or dissociates more easily from) the enzyme. Furthermore, the charge transfer complex formed between MLCADH with 3-thiaoctanoyl-CoA is less stable compared to that between MCADH with the substrate analog (Nandy et al., 1996). Again, the stability of the charge transfer complex is probably directly related to the binding energy of the analog to the active site of the enzyme. The binding energy between the ligand and the enzyme must be greater in the wild type than in MLCADH due to the van der Waal's interactions caused by the tight fitting at the active site (Figure 7). In addition, in the case of 3-thiaoctanoyl$\mathrm{CoA}$, solvent molecules in the active site of the mutant enzyme destabilyze the enolate charge transfer donor formed by the deprotonation at the $\mathrm{C}_{\alpha}$ position of the analog.

Conclusion. We confirm that, despite the different position in their primary amino acid sequences, the catalytic base in acyl-CoA dehydrogenases is topologically conserved in the three-dimensional structure. Furthermore, our structural studies together with the kinetic studies of MLCADH in the preceding paper (Nandy et al., 1996) demonstrate that the location of the catalytic base in the active site can alter substrate chain length specificity of MCADH by modulating the distance and orientation of the base with respect to the $\mathrm{C}_{\alpha}$ proton to be abstrated. Although an example of "jumping catalytic residue" has been reported in the lipase family (Schrag et al., 1992), our studies appear to be the first illustration of two functional groups, extending from two different segments of an enzyme, carrying out exactly the same chemistry of catalysis.

\section{ACKNOWLEDGMENT}

We thank Dr. David Roberts for calculating solvent accessible areas and generating Figures 5 and 7.

\section{REFERENCES}

Ankele, K., Melde, K., Engst, S., Bross, P., Ghisla, S., \& Strauss, A. W. (1991) in Flavins and Flavoproteins: Proceedings of the Tenth International Symposium, Como (Italy) (Curti, B., Ronchi, S., \& Zanetti, G., Eds.) pp 325-328, Walter de Gruyter \& Co., Berlin.
Aoyama, T., Ueno, I., Kamijo, T., \& Hashimoto, T. (1994) J. Biol. Chem. 269, 19088-19094.

Beinert, H. (1963) in The Enzymes, 2nd ed. (Bpyer, P. D., Lardy, H., \& Myrback, K., Eds.) Vol. 7, pp 447-466, Academic Press, New York.

Bross, P., Engst, S., Strauss, A. W., Kelly, D. P., Rasched, I., \& Ghisla, S. (1990) J. Biol. Chem. 265, 7116-7119.

Brunger, A. (1992) X-PLOR, Version 3.1, Yale University, New Haven, CT.

Cambilau, C., \& Roussel, A. (1993) TURBO-FRODO Manual, Version 4.2, Bio-Graphics, Marseille, France.

Connolly, M. (1983) Science 221, 709-713.

Djordjevic, S., Dong, Y., Paschke, R., Frerman, F. E., Strauss, A. W., \& Kim, J. J. P. (1994) Biochemistry 33, 4258-4264.

Gandour, R. D. (1981) Bioorg. Chem. 10, 169-176.

Ghisla, S., Engst, S., Moll, M., Bross, P., Strauss, A. W., \& Kim, J.-J. P. (1992) in New Develpments in Fatty Acid Oxidation (Coates, P. M., \& Tanaka, K., Eds.) pp 127-142, Wiley-Liss, New York.

Gregersen, N., Winter, V., Curtis, D., Deufel, T., Mack, M., Hendrickx, J., Willems, P. J., Ponzone, A., Parrella, T., Ponzone, R., Ding, J. H., Zhang, W., Chen, Y. T., Kahler, S., Roe, C. R., Kølvraa, S., Schneiderman, K., Andresen, B. S., Bross, P., \& Bolund, L. (1993) Hum. Hered. 43, 342-350.

Higashi, T. (1990) J. Appl. Crystallogr. 23, 253-257.

Joseph-McCarthy, D., Rost, L. E., Komivies, E. A., \& Petsko, G. A. (1994) Biochemistry 33, 2824-2829.

Kim, J. J. P., Wang, M., \& Paschke, R. (1993) Proc. Natl. Acad. Sci. U.S.A. 90, 7523-7527.

Kim, J. J. P., Wang, M., Djordjevic, S., Paschke, R., Bennett, D. W., \& Vockley, J (1994) in Flavins and Flavoproteins, Proceddings of the Eleventh International Symposium, Nagoya, Japan (Yagi, K., Ed.) pp 273-282, Walter de Gruyter \& Co., Berlin.

Lau, S.-M., Brantley, R. K., \& Thorpe, C. (1988) Biochemistry 27, 5089-5095.

Lenich, A. C., \& Goodman, S. I. (1986) J. Biol. Chem. 291, 40904096.

Matsubara, Y., Ito, M., Glassberg, R., Satyabhama, S., Ikeda, Y., \& Tanaka, K. (1990) J. Clin. Invest. 85, 1058-1064.

McPherson, A. (1985) Methods Enzymol. 114, 112-128.

Mohsen, A.-W., \& Vockley, J. (1995) Biochemistry 34, 1014610512.

Nandy, A., Kieweg, V., Kräutle, F.-G., Vock, P., Küchler, B., Bross, P., Kim, J.-J. P., Rasched, I., \& Ghisla, S. (1996) Biochemistry $35,12402-12411$

Powell, P. J., \& Thorpe, C. (1988) Biochemistry 27, 8022-8028.

Sawyer, L., \& James, M. N. G. (1982) Nature 295, 79-80.

Schrag, J. D., Winkler, F. K., \& Cygler, M. (1992) J. Biol. Chem. 267, 4300-4303.

Srivastava, D. K., Kumar, N. R., \& Peterson, K. (1995) Biochemistry $34,4625-4632$.

Tanaka, K., \& Indo. Y. (1992) in New Develpments in Fatty Acid Oxidation (Coates, P. M., \& Tanaka, K., Eds) pp 95-110, WileyLiss, New York.

Trievel, R. C., Wang, R., Anderson, V. E., \& Thorpe, C. (1995) Biochemistry 34, 8597-8605.

Wang, R., \& Thorpe, C. (1991) Biochemistry 30, 7895-7901. 